\title{
THE PROJECTIVE CLASS GROUP OF THE FUNDAMENTAL GROUP OF A SURFACE IS TRIVIAL ${ }^{1}$
}

\author{
KOO-GUAN CHOO
}

\begin{abstract}
Let $D=F_{1} \times F_{2} \times \cdots \times F_{n}$ be a direct product of $n$ free groups $F_{1}, F_{2}, \cdots, F_{n}, \alpha$ an automorphism of $D$ which leaves all but one of the noncyclic factors in $D$ pointwise fixed and $T$ an infinite cyclic group. Let $D \times_{\alpha} T$ be the semidirect product of $D$ and $T$ with respect to $\alpha$. We prove that the Whitehead group of $D \times{ }_{\alpha} T$ and the projective class group of the integral group ring $Z\left(D \times{ }_{\alpha} T\right)$ are trivial. The second result implies that the projective class group of the integral group ring over the fundamental group of a surface is trivial.
\end{abstract}

Let $G$ be a group. We denote the Whitehead group of $G$ by Wh $G$ and the projective class group of the integral group ring $Z(G)$ of $G$ by $\widetilde{K}_{0} Z(G)$. Let $\alpha$ be an automorphism of $G$ and let $T$ be an infinite cyclic group. Then we denote by $G \times{ }_{\alpha} T$ the semidirect product of $G$ and $T$ with respect to $\alpha$.

Let $M$ be a connected 2-dimensional manifold and $\pi_{1}(M)$ the fundamental group of $M$. If $M$ is open, then $\pi_{1}(M)$ is a free group so that $\tilde{K}_{0} Z\left(\pi_{1}(M)\right)$ is trivial by a theorem of Bass (cf. [1]). Next, if $M$ is a sphere or a projective plane, then $\pi_{1}(M)=0$ or $T_{2}$ (cyclic group of order 2) and so $\tilde{K}_{0} Z\left(\pi_{1}(M)\right)=0$ (cf. [7, p. 419]). Now, if $M$ is closed and is not a sphere or projective plane, then Farrell-Hsiang [4] have shown that $\pi_{1}(M)$ is just the semidirect product $F \times{ }_{\alpha} T$, where $F$ is a free group. The purpose of this paper is to show that $\widetilde{K}_{0} Z\left(F \times{ }_{\alpha} T\right)=0$ and so the projective class group of the integral group ring over the fundamental group of a surface is always trivial. In fact, we prove:

Received by the editors December 4, 1972.

AMS (MOS) subject classifications (1970). Primary 13D15, 18F25, 55A05; Secondary 57C20, 57D80.

Key words and phrases. Projective class group, Whitehead group, fundamental group, direct product of free groups.

${ }^{1}$ This research was supported in part by a postgraduate fellowship of the National Research Council of Canada. It contains parts of the results from the author's doctoral thesis at the University of British Columbia written under the direction of Professor E. Luft. The author is most indebted to Professor E. Luft, and to Professor K. Y. Lam for their valuable suggestions and encouragement during the preparation of the thesis.

(c) American Mathematical Society 1973 
THEOREM A. Let $D=F_{1} \times F_{2} \times \cdots \times F_{n}$ be a direct product of $n$ free groups $F_{1}, F_{2}, \cdots, F_{n}$ and $\alpha$ an automorphism of $D$ which leaves all but one of the noncyclic factors in $D$ pointwise fixed. Then $\tilde{K}_{0} Z\left(D \times_{\alpha} T\right)=0$.

When $n=1$, this implies the result mentioned above.

In [4], Farrell-Hsiang also proved that $\mathrm{Wh}\left(F \times_{\alpha} T\right)=0$. We will generalize it to:

THEOREM B. Let $D$ and $\alpha$ be as given in Theorem A. Then $\mathrm{Wh}\left(D \times_{\alpha} T\right)=$ 0 .

To prove Theorem $B$, we need the following:

Lemma 1. Let $D$ be as given in Theorem A. Then Wh $D=0$ and $\tilde{K}_{0} Z(D)=0$.

Before proving Lemma 1, we recall some definitions and results from [4] and [3]. For more details, we refer to [4] and [3].

Let $R$ be an associative ring with identity and $\alpha$ an automorphism of $R$. We denote by $R_{\alpha}[T]$, the $\alpha$-twisted finite Laurent series ring of $R$. Recall that an additive map $\phi$ from a right $R$-module $M_{1}$ to a right $R$-module $M_{2}$ is $\alpha$-linear if $\phi(m r)=\phi(m) \alpha(r)$ for $m \in M_{1}$ and $r \in R$. Let $\mathscr{C}(R, \alpha)$ be the category whose objects are pairs $(P, \phi)$ where $P$ is a finitely generated projective right $R$-module and $\phi$ is an $\alpha$-linear nilpotent endomorphism of $P$, and whose morphisms $g:\left(P_{1}, \phi_{1}\right) \rightarrow\left(P_{2}, \phi_{2}\right)$ are $R$-linear homomorphisms $g: P_{1} \rightarrow P_{2}$ such that the following diagram

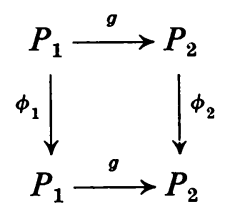

is commutative. Let $C^{\prime}(R, \alpha)$ be the Grothendieck group of the category $\mathscr{C}(R, \alpha)$. The class of an element $(P, \phi) \in \mathscr{C}(R, \alpha)$ in $C^{\prime}(R, \alpha)$ is denoted by $[P, \phi]$. The class $[R, 0]$ generates a cyclic subgroup $F(R)$ of $C^{\prime}(R, \alpha)$. Let $C(R, \alpha)=C^{\prime}(R, \alpha) / F(R)$ and let $\widetilde{C}(R, \alpha)$ be the subgroup of $C(R, \alpha)$ generated by $\left[R^{n}, \phi\right]$ for $\left(R^{n}, \phi\right) \in \mathscr{C}(R, \alpha)$. For any abelian group $G$ with an automorphism $\beta$, let $G^{\beta}=\{g \mid \beta(g)=g, g \in G\}$, and let $I(\beta)=$ $\{g-\beta(g) \mid g \in G\}$. Then we have:

THEOREM 1 ([4, THEOREM 19] AND [2]) (Farrell-Hsiang decomposition formula for $K_{1} R_{\alpha}[T]$; Bass-Heller-Swan decomposition formula when $\alpha=$ identity).

$$
K_{1} R_{\alpha}[T] \cong X \oplus \widetilde{C}(R, \alpha) \oplus \widetilde{C}\left(R, \alpha^{-1}\right)
$$


and $X$ is given by the following exact sequence

$$
0 \rightarrow K_{1} R / I\left(\alpha_{*}\right) \rightarrow X \rightarrow\left(K_{0} R\right)^{\alpha_{*}} \rightarrow 0,
$$

where $\alpha_{*}$ is the automorphism of $K_{1} R$ (resp. $\left.K_{0} R\right)$ induced by $\alpha$.

THEOREM 2 ([4, TheOREM 21] AND [2]). (Farrell-Hsiang decomposition formula for $\mathrm{Wh}\left(G \times{ }_{\alpha} T\right)$; Bass-Heller-Swan decomposition formula when $\alpha=$ identity).

$$
\mathrm{Wh}\left(G \times_{\alpha} T\right) \cong X \oplus \widetilde{C}(Z(G), \alpha) \oplus \widetilde{C}\left(Z(G), \alpha^{-1}\right)
$$

and $X$ is given by the following exact sequence

$$
0 \rightarrow \text { Wh } G / I\left(\alpha_{*}\right) \rightarrow X \rightarrow\left(\tilde{K}_{0} Z(G)\right)^{\alpha_{*}} \rightarrow 0 .
$$

Now, we recall that a ring $R$ is right coherent if any homomorphism $f: R^{n} \rightarrow R^{m}$ has finitely generated kernel. In [5], Farrell has shown that if $R$ is right regular, then $\widetilde{C}(R, \alpha)=0$ for any automorphism $\alpha$ of $R$. But, in [3], Choo, Lam and Luft have pointed out that if $R$ is right coherent and has finite right global dimension, then $\tilde{C}(R, \alpha)=0$ for any automorphism $\alpha$ of $R$. This result implies, for example, that for a group $G$ with $Z(G)$ right coherent and of finite right global dimension, the exotic summand $\widetilde{C}(Z(G), \alpha)$ in the Farrell-Hsiang decomposition formula for $\mathrm{Wh}\left(G \times{ }_{\alpha} T\right)$ becomes zero. Moreover, they proved, in particular, that for any free group $F$ and for any abelian group $A, Z(A \times F)$ is right coherent.

Next, let $R(G)$ be the group ring of $G$ over a ring $R$. We denote the cokernel of the homomorphism $K_{1} R \rightarrow K_{1} R(G)$ (induced by the inclusion $R \rightarrow R(G)$ ) by $R_{1} R(G)$. The following result is an immediate consequence of [8, Theorem 6.2].

THEOREM 3. Let $R$ be a ring such that $\widetilde{C}(R$, id $)=0$ and let $G_{1}$ and $G_{2}$ be groups. Then $R_{1} R\left(G_{1} * G_{2}\right) \cong R_{1} R\left(G_{1}\right) \oplus R_{1} R\left(G_{2}\right)$. In particular, if $F$ is a free group of rank $m$, we write $F=T_{1} * T_{2} * \cdots * T_{m}$ as a free product of $m$ infinite cyclic groups, so that

$$
R_{1} R(F) \cong \bigoplus_{j=1}^{m} R_{1} R\left(T_{j}\right)
$$

Proof of Lemma 1. Let the number of noncyclic factors in $D$ be $k$. We will prove the lemma by induction on $k$.

For $k=0, D$ is just a free abelian group so that Wh $D=0$ and $\tilde{K}_{0} Z(D)=0$ (cf. [2]). This starts the induction.

Now, suppose inductively that the lemma holds for any such group with $k-1$ noncyclic factors. To show Wh $D=0$, write $D=D^{\prime} \times F$ where $F$ is noncyclic, and the number of noncyclic factors in $D^{\prime}$ is $k-1$. Then for any infinite cyclic group $T, \mathrm{Wh}\left(D^{\prime} \times T\right)=0$ by induction hypothesis. It follows 
from Theorem 2 that

$$
\widetilde{C}\left(Z\left(D^{\prime}\right), \mathrm{id}\right)=0 \text { and } \tilde{K}_{0} Z\left(D^{\prime}\right)=0 .
$$

Next, suppose that $F$ is of finite rank $m$ and write $F=T_{1} * T_{2} * \cdots * T_{m}$ as a free product of $m$ infinite cyclic groups. Since $\widetilde{C}\left(Z\left(D^{\prime}\right)\right.$, id $)=0$, it follows from Theorem 3 that

$$
R_{1} Z\left(D^{\prime}\right)(F) \cong \bigoplus_{j=1}^{m} \bar{K}_{1} Z\left(D^{\prime}\right)\left(T_{j}\right)
$$

Again, using $\widetilde{C}\left(Z\left(D^{\prime}\right)\right.$, id $)=0$, we deduce from Theorem 1 that the sequence $0 \rightarrow K_{1} Z\left(D^{\prime}\right) \rightarrow K_{1} Z\left(D^{\prime}\right)\left(T_{j}\right) \rightarrow K_{0} Z\left(D^{\prime}\right) \rightarrow 0$ is short exact for each $j$; i.e., $R_{1} Z\left(D^{\prime}\right)\left(T_{j}\right)=K_{0} Z\left(D^{\prime}\right)$ for each $j$. Hence, it follows from (2) that $R_{1} Z\left(D^{\prime}\right)(F) \cong K_{0} Z\left(D^{\prime}\right) \oplus \cdots \oplus K_{0} Z\left(D^{\prime}\right)$ ( $m$ copies); i.e., the sequence

$$
0 \rightarrow K_{1} Z\left(D^{\prime}\right) \rightarrow K_{1} Z\left(D^{\prime}\right)(F) \rightarrow K_{0} Z\left(D^{\prime}\right) \oplus \cdots \oplus K_{0} Z\left(D^{\prime}\right) \rightarrow 0
$$

is short exact. Passing to Whitehead groups, we have (cf. [4])

$$
0 \rightarrow \mathrm{Wh} D^{\prime} \rightarrow \mathrm{Wh}\left(D^{\prime} \times F\right) \rightarrow \tilde{K}_{0} Z\left(D^{\prime}\right) \oplus \cdots \oplus \tilde{K}_{0} Z\left(D^{\prime}\right) \rightarrow 0 .
$$

Hence, by (1) and the induction hypothesis for $D^{\prime}$, we have Wh $D=$ $\mathrm{Wh}\left(D^{\prime} \times F\right)=0$.

The case when $F$ has infinite rank does not need to worry us since a matrix over $Z\left(D^{\prime}\right)(F)$ involves entries which are sums of words involving only a finite number of free generators of $F$.

Finally, it follows from Theorem 2 and the triviality of $\mathrm{Wh}(D \times T)$ that $\tilde{K}_{0} Z(D)=0$. This completes the proof.

We remark that the first result in Lemma 1 extends that of Bass-HellerSwan [2] for free abelian groups and of Gersten-Stallings [8] for free groups; and the other extends that of Bass-Heller-Swan [2] for free abelian groups, of Bass [1] for free groups and of Gersten [6] for direct product of a free abelian group and a free group.

Proof of TheOREM B. Let $k$ be the number of noncyclic factors in $D$. We prove by induction on $k$.

For $k=0, D$ is just a free abelian group $A$ and so, by Theorem 2 , $\mathrm{Wh}\left(D \times{ }_{\alpha} T\right)=0$ since $\tilde{C}(Z(A), \alpha)=0$, Wh $A=0$ and $\tilde{K}_{0} Z(A)=0$ (cf. [2]).

For $k=1, D$ is of the form $A \times F$ with $A$ free abelian of finite rank and $F$ noncyclic. Then, in the Farrell-Hsiang decomposition formula for $\mathrm{Wh}\left((A \times F) \times{ }_{\alpha} T\right)$ (cf. Theorem 2), the terms Wh $(A \times F)$ and $\tilde{K}_{0} Z(A \times F)$ are trivial by Lemma 1 . Also, thanks to the coherence property of $Z(A \times F)$ and the fact that $Z(A \times F)$ is of finite right global dimension, $\widetilde{C}(Z(A \times F), \alpha)=0$ (cf. [3]). Hence Wh $\left(D \times{ }_{\alpha} T\right)=\mathrm{Wh}\left((A \times F) \times_{\alpha} T\right)=0$.

Now, suppose inductively that the theorem holds for any such group with $k-1$ nonclyclic factors. Let $D=H \times F$ with $F$ noncyclic and $\alpha$ fixed 
on $F$ while $H$ has $k-1$ noncyclic factors. To show that $\operatorname{Wh}\left(D \times{ }_{\alpha} T\right)=0$, write $D \times_{\alpha} T=D^{\prime} \times F$ with $D^{\prime}=H \times_{\alpha} T$. The situation is now completely analogous to Lemma 1 and the same argument there gives $\mathrm{Wh}\left(D \times{ }_{\alpha} T\right)=$ 0 . This completes the proof.

Proof of Theorem A. Consider the group $\left(D \times{ }_{\alpha} T\right) \times T_{1}$, where $T$ and $T_{1}$ are infinite cyclic groups. This is just the group $\left(D \times T_{1}\right) \times_{\alpha \times i d T_{1}} T$. By Theorem B, Wh $\left(\left(D \times T_{1}\right) \times_{\alpha \times \mathbf{i d} T_{1}} T\right)=0$ so that $\mathrm{Wh}\left(\left(D \times{ }_{\alpha} T\right) \times T_{1}\right)=$ 0 . The result now follows from Theorem 2 . This completes the proof.

Finally, we note that there is a topological application of Theorem B. If $M$ is a closed surface (other than the real projective plane) and $\left(S^{1}\right)^{k}$ is the $k$-dimensional torus, then the fundamental group of $M \times\left(S^{1}\right)^{k}$ is of the form $D \times_{\alpha} T$. Hence, the triviality of $\mathrm{Wh}\left(D \times_{\alpha} T\right)$ implies (cf. [7, p. 393]).

COROLlaRY. If $N$ is a differentiable or PL manifold of $\operatorname{dim} \geqq 5$ which is $h$-cobordant to $M \times\left(S^{1}\right)^{k}$, where $M$ is a closed surface, then $N$ is actually diffeomorphic or PL-homeomorphic to $M \times\left(S^{1}\right)^{k}$ respectively.

\section{REFERENCES}

1. H. Bass, Projective modules over free groups are free, J. Algebra 1 (1964), 367-373. MR 31 \#2290.

2. H. Bass, A. Heller and R. G. Swan, The Whitehead group of a polynomial extension, Inst. Hautes Études Sci. Publ. Math. No. 22 (1964), 61-79. MR 30 \#4806.

3. K. G. Choo, K. Y. Lam and E. Luft, On free product of rings and the coherence property, Proc. Conf. on Algebraic $K$-Theory (Seattle, 1972) (to appear).

4. F. T. Farrell and W. C. Hsiang, A formula for $K_{1} R_{\alpha}[T]$, Proc. Sympos. Pure Math., vol. 17, Amer. Math. Soc., Providence, R.I., 1970, pp. 192-218. MR 41 \#5457.

5. F. T. Farrell, The obstruction of fibering a manifold over a circle, Indiana Univ. Math. J. 21 (1971), 315-346.

6. S. Gersten, On class groups of free products, Ann. of Math. (2) 85 (1968), 392-398. MR 37 \#254.

7. J. Milnor, Whitehead torsion, Bull. Amer. Math. Soc. 72 (1966), 358-426. MR 33 \#4922.

8. J. Stallings, Whitehead torsion of free products, Ann. of Math. (2) 82 (1965), 354-363. MR 31 \#3518.

Department of Mathematics, University of British Columbia, Vancouver 8, B.C., Canada 